\title{
Experimental analysis of fabric substrate on a moisture sensor
}

\author{
Marc Martínez-Estrada*, Raúl Fernández-García, Ignacio Gil. \\ Department of Electronic Engineering, Universitat Politècnica de Catalunya, \\ c/Colom n ${ }^{\circ}$ 1-3 TR2 08222, Spain. \\ E-mail: marc.martinez.estrada@upc.edu
}

Received xxxxxx

Accepted for publication $\mathrm{xxxxxx}$

Published xxxxxx

\begin{abstract}
In this work, an embroidered textile moisture sensor is proposed and characterized with three different fabric substrates. The sensors are based on a capacitive interdigitated structure embroidered on a textile substrate with a conductive yarn. The performance of three different type of substrates has been addressed. In order to evaluate the sensor behavior, the impedance of the sensor has been measured by means of an LCR meter from $20 \mathrm{~Hz}$ to $200 \mathrm{kHz}$ on a climatic chamber with a sweep of the relative humidity from $30 \%$ to $80 \%$ at $20^{\circ} \mathrm{C}$. The results show that the fabric substrate defines the electrical properties of the sensor due to both, the substrate permittivity and size yarn thickness increase. This sensor dependence determines the optimum substrate to be used to develop wearable applications for moisture measurement.
\end{abstract}

Keywords: Sensor; e-textile; embroidery; moisture; capacitive; substrate

\section{Introduction}

Nowadays, the interest in the development of wearable sensors for biological sensing applications is increasing. These onbody sensors are suitable for applications such as health monitoring[1,2], physical training [3,4], emergency rescue service and law-enforcement [5]. In order to implement these sensors, textile substrates are selected as the most convenient choice in the development of wearable electronic applications. This is due to the fact that humans have been covering their body with fabrics through the years, which makes fabrics the most suitable substrate to integrate on-body sensors and other electronic components. The integration of sensors in textiles can be carried out by different techniques, such as ink-jet printing, screen printing, stamp transfer, electrospinning, dip coating[6], embroidery or conductive yarns introduction in the manufacturing process $[7,8,9,10]$. Embroidery has been revealed as the most cost-effective technique to implement wearable sensors into fabrics. This optimal balance is due to the availability of the manufacturing technology (industrial embroidery machines), the efficient exploitation of the expensive specialized conductive threads and the repeatability of geometries and layouts [11]. Furthermore, the possibility of achieving prototypes mass production is an advantage to select the manufacturing process for the sensors in terms of overall production cost. Moreover, in contrast to other manufacturing processes, like ink-jet-printing, embroidered do not require any mask or chemical product. This fact implies direct impact on the production time and costs [12].

Nowadays the possibilities that embroidered sensors can provide are unlimited. There are a lot of different substrates where a sensor could be embroidered on to gather data from the environment or the human body [13]. The two most used materials for textiles are cotton and polyethylene terephthalate $[14,15,16]$. Cotton is a natural staple fiber that grows around the seeds of cotton plants. This material is manufactured to produce spun yarns. The polyethylene terephthalate, commonly known as Polyester (PES) is used to be built in filament or multifilament yarns.

Despite of both textile materials can be used to develop any type of clothing, polyester textile production is mainly focused on fields like sport and fitness due to its properties, whereas the cotton applications are usually focused on personal comfort 
and it is commonly used in daily clothes. Considering such different applications, the aim of the work is to analyze the impact of several substrates on the sensor behavior and to assess if the substrates themselves could determine the final application of the sensor. To achieve these goals, in this paper, a comparison of moisture interdigitate sensor behavior with three different substrates will be experimentally analyzed with a fixed layout pattern and conductive yarn sensor as well as constant manufacturing settings in all the cases.

Embroidered sensors provides a great opportunity to introduce sensors in places where before was inimaginable. As a example sensor could being introduced in jackets or trousers where the users could have date from their sorrounding. Maybe this application is more interesting when a policeman or a firefighter could have the sensor in their clothes and data could help them in their daywork. Other places where the sensor could be introduced are home textiles. Sensor could be installed in beds or curtains and provide the user information abaout the home enviorment or the bed state. For exameple, for a young child that is learning to not urinate on bed at night.

\section{Methods and materials}

The proposed moisture sensor is based on a capacitive embroidered interdigitated structure whose dimensions are depicted in Figure 1. In order to embroider the sensor on the substrates under analysis, a commercial Shieldex 117/17 2-ply has been chosen. This conductive yarn is made by a coating process, where a filament of polyamide (PA) is coated with pure silver. After the yarn is defined, it is needed to select the different substrates under test. In this investigation a cotton woven fabric (CO), a polyester woven fabric (PES) and a medical cotton woven fabric (MED) were selected for the sensor characterization tests. All the textiles are variations of plain weave structure. The properties of the different fabrics are summarized in Table 1 and the sensors are showed on Figure 2.

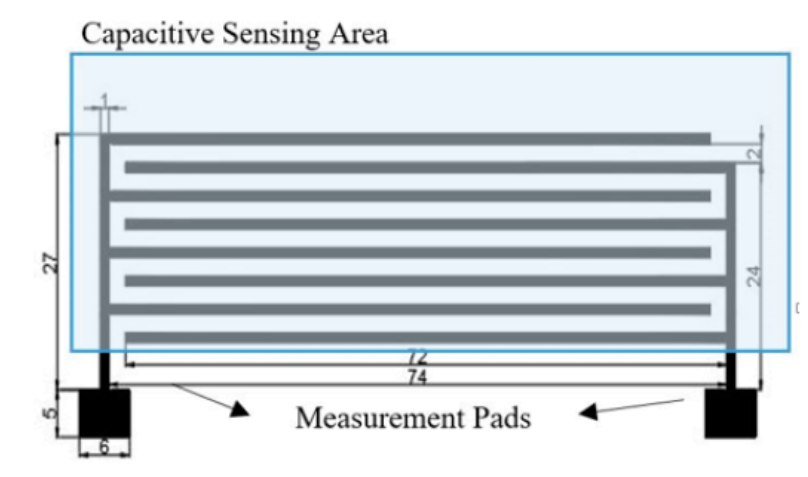

Figure 1. Layout of the embroidered sensor (dimensions in $\mathrm{mm}$ )

Table 1. Substrate properties

\begin{tabular}{ccccc}
\hline Substrates & $\begin{array}{c}\text { Thickness } \\
(\mathbf{m m})\end{array}$ & Permittivity & $\begin{array}{c}\text { Loss } \\
\text { tangent }\end{array}$ & $\begin{array}{c}\text { Moisture } \\
\text { regains } \\
\text { (Weight \%) }\end{array}$ \\
\hline $\begin{array}{c}\text { Cotton } \\
(\mathrm{CO})\end{array}$ & 0.256 & 1.87 & 0,0750 & $5.96 \%$ \\
$\begin{array}{c}\text { Medical cotton } \\
\text { (MED) }\end{array}$ & 0.306 & 2.02 & 0.0671 & $8.02 \%$ \\
$\begin{array}{c}\text { Polyester } \\
\text { (PES) }\end{array}$ & 0.199 & 1.5 & 0.0189 & $2.23 \%$ \\
\hline
\end{tabular}

Cotton and polyester yarns react in different ways under humidity conditions. In fact, cotton is more affected by the changes of humidity, due to its hydrophilic nature. In the case of the polyester, its hydrophobic nature reduces the impact of the moisture in the textile feature. Both cotton textiles have being more affected by humidity than polyester substrate, but they still have differences between them. The main differences between cotton fabric and medical cotton fabric are two. On the one side, the yarn used for the medical cotton fabric is more porous than the yarn used on the cotton fabric, on the other side the medical 
cotton fabric has an antibacterial chemical treatment. Therefore, different sensor behavior should be expected when these fabrics are used as humidity sensor substrates.

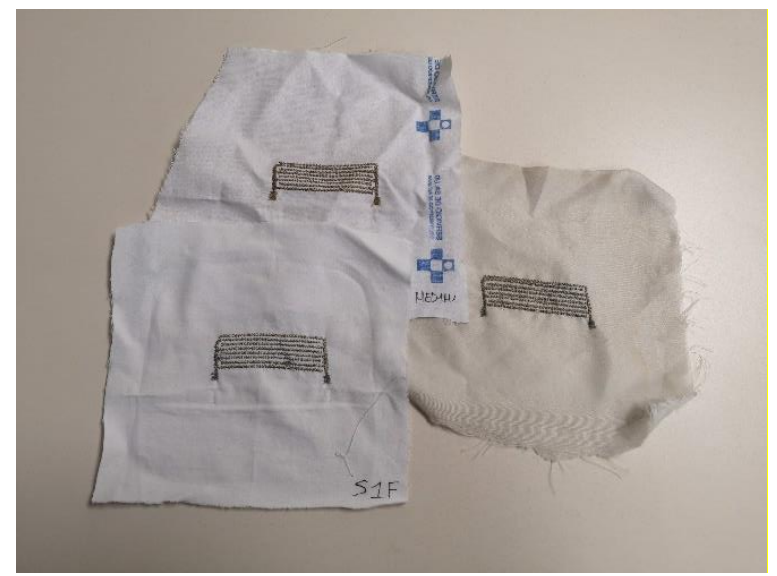

Figure 2. Sensors embroidered on different substrates. Left bottom: Cotton fabric. Left top: Medical cotton fabric. Right: Polyester fabric.

To embroider the pattern in all the considered substrates, a Singer Futura XL-550 embroidery machine is used. The properties of the layout pattern are the same for all the manufactured sensors embroidered. They are embroidered with a satin fill stitch in order to achieve a homogeneous yarn distribution over the sensor surface, the integrated process is represented at Figure 3. The differences between the thicknesses of the substrates make not necessary a different machine set up. In order to achieve the best comfort and reduce the conductive yarn cost, a support cotton yarn has been used. At the end of the manufacturing process it is observed that the sensors have in one side conductive yarn and in the other side the cotton yarn. In order to experimentally compare the sensors' response under different humidity points, they have been tested in a CCK-25/48 Dycometal climatic chamber. The sensors impedances have been measured by means of an external Rhode \& Schwarz HM8118 LCR meter. An image of the experimental setup is shown in Figure 4.

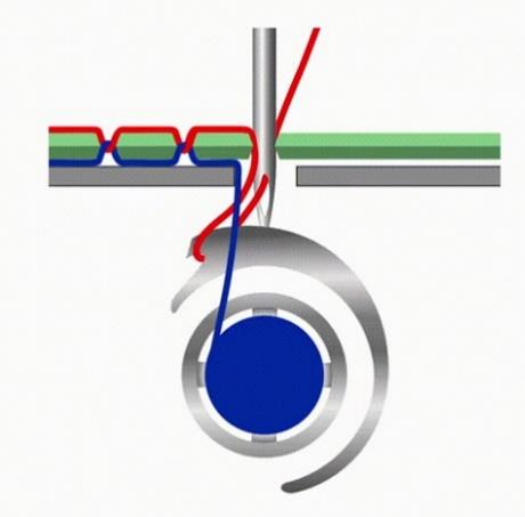

Figure 3. Embroidery process to integrate the sensor on the substrate[17]

The sensor impedances, which corresponds to the measurement of the opposition of the sensor structure presents to current when a voltage is applied, have been measured from $20 \mathrm{~Hz}$ to $200 \mathrm{kHz}$ in a $30 \%$ to $80 \%$ range of relative humidity (RH) environment, whereas the temperature has remained constant at $20^{\circ} \mathrm{C}$. Higher impedance shifts better sensor achievement. In addition, a second test was done with the objective of analyzing the permittivity performance against the relative humidity, which is a measure of the electric polarizability of a dielectric. For this test a split post dielectric resonator (SPDR, QWED) with a Microwave Frequency Q-Meter has been used. (Figure 2b). 


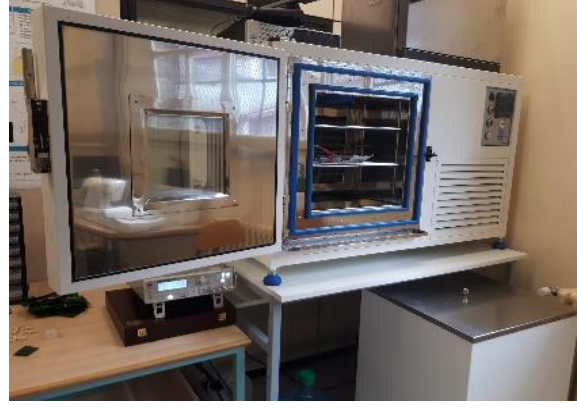

(a)

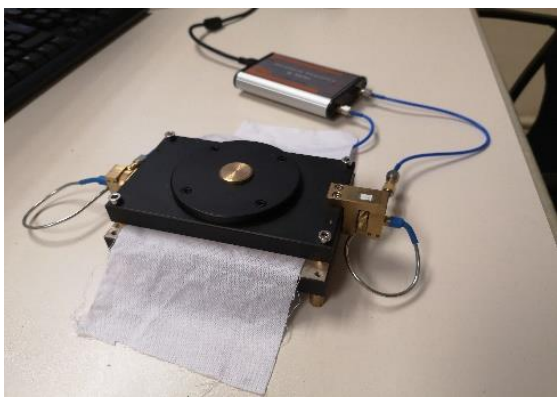

(b)

Figure 4. (a) Image of the experimental setup for humidity sensor testing. (b) Test of the permittivity and loss tangent of the textile samples based on SPDR

\section{Results and discussion}

Figure 5 and Figure 6 show the experimental impedance frequency response for each substrate under investigation at $40 \%$ RH and $80 \%$ RH, respectively. It can be observed how the impedance sensor frequency response is affected by both, the substrate material and the relative humidity. More specifically, at $40 \% \mathrm{RH}$, the impedance module decreases with the frequency in all cases. However, the impedance reduction is different depending on the substrate material. Medical Cotton module (MED) impedance varies from $6.6 \mathrm{M} \Omega$ to $29 \mathrm{k} \Omega$, standard cotton $(\mathrm{CO})$ varies from $176 \mathrm{M} \Omega$ to $40 \mathrm{k} \Omega$ and finally polyester (PES) vary from $1.19 \mathrm{G} \Omega$ to $41 \mathrm{k} \Omega$. At low frequency $(<100 \mathrm{~Hz})$ the MED substrate shows a resistive impedance, whereas the PES substrate shows a capacitive behavior. At higher frequency the phase impedance in all sensors trend to $-90^{\circ}$, pointing out the capacitive behavior in all cases.

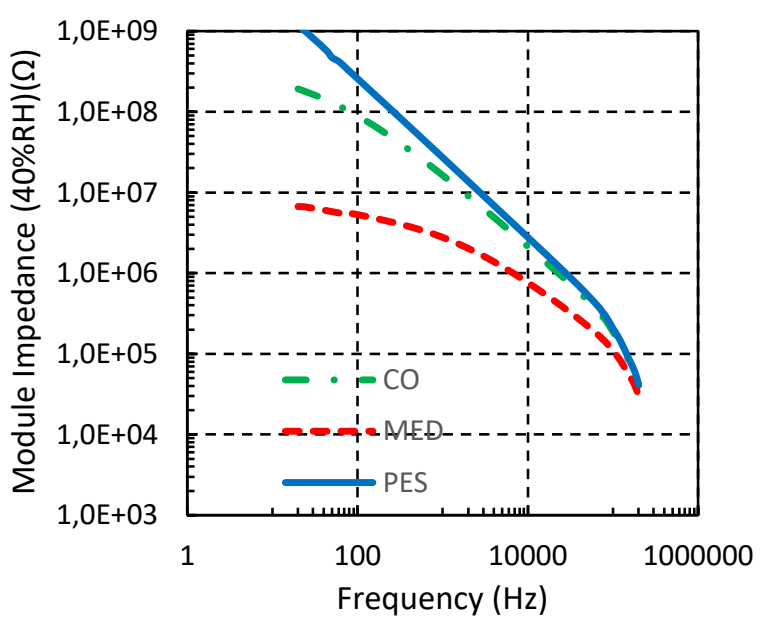

b) Impedance module at $40 \% \mathrm{RH}$

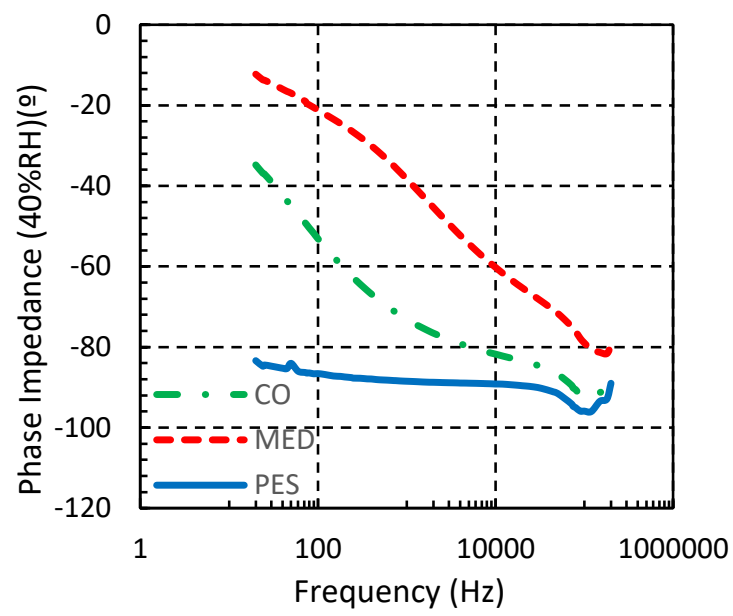

b) Impedance phase at $40 \% \mathrm{RH}$

Figure 5. Experimental frequency response of sensor impedance at $40 \% \mathrm{RH}$ 


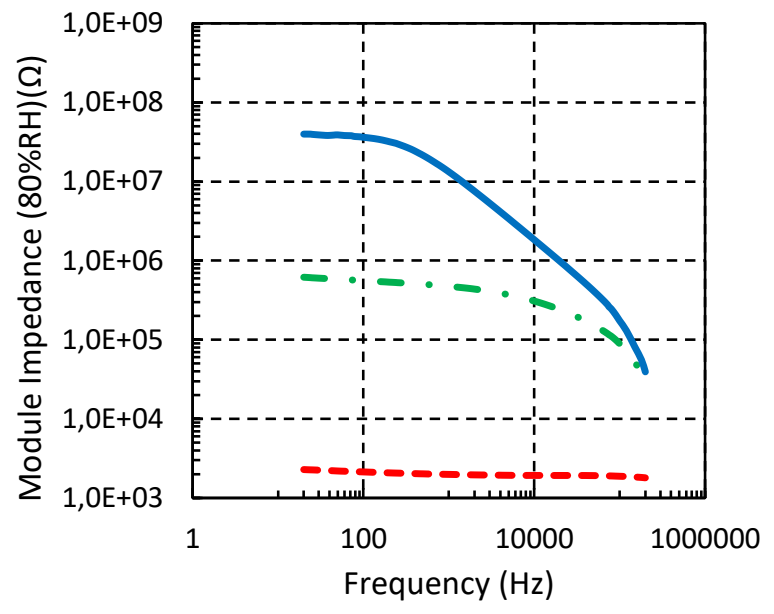

a) Impedance module at $80 \% \mathrm{RH}$

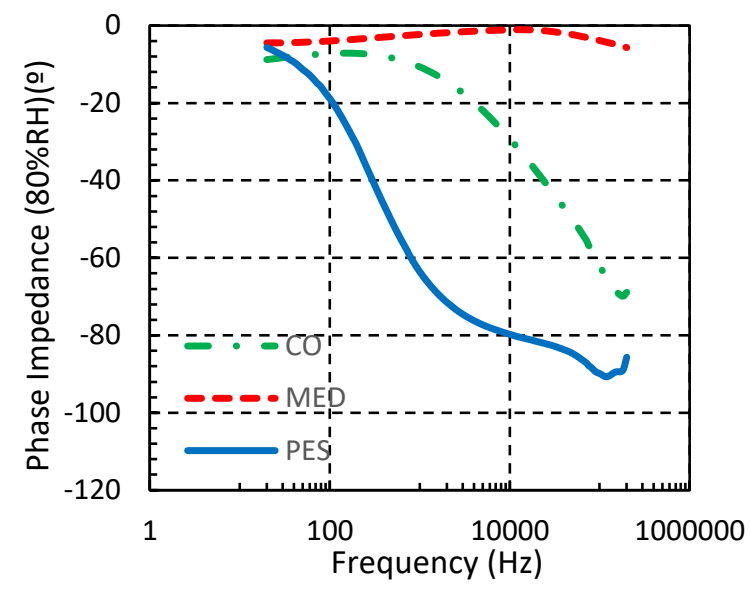

b) Impedance phase at $80 \% \mathrm{RH}$

Figure 6. Experimental frequency response of sensor impedance at $80 \% R H$

If the moisture is increased up to $80 \% \mathrm{RH}$ (Figure 6) a clear difference on the sensor frequency response is measured. In particular, for MED substrate the sensor impedance is almost constant in terms of the frequency with a value of about $2 \mathrm{k} \Omega$ and the phase is close to 0 degree. These results denote that MED substrate at this moisture level behaves as an effective resistor. In contrast, the PES and CO sensors work as a resistance-capacitive (RC) circuit with an impedance reduction from $39 \mathrm{M} \Omega$ to $0.6 \mathrm{M} \Omega$ for the PES substrate and from $609 \mathrm{k} \Omega$ to $20 \mathrm{k} \Omega$ for the CO substrate. The higher MED substrate conductivity can be explained by means of the chemical treatment that it is normally applied to medical fabrics which decreases the substrate parasitic resistivity and, as a result, it is more affected by the relative humidity. The chemical treatment applied to that kind of medical fabric is an antibacterial treatment, which consist in a chemical process that increrase the conductivity of the fabric to prevent the fabric from bacterias, it has a similar behaviour as a silver yarn being introduced onto the fabric. In the polyester case the behavior is like it was expected. Its hydrophobic nature implies that the polyester is less affected by relative humidity. In fact, the humidity remains on the surface of the fabric and there is no absorption.

In order to characterize the sensors, the moisture has been range from 30 to $80 \% \mathrm{RH}$ at $2 \mathrm{kHz}$. This frequency has been chosen for being one of the most suitable frequency to be used, which gives a desirable module and phase impedance values for the humidity range that can be easily measured with a low cost integrated circuit, such as the Texas Instrument AD5933 impedance converter [18].

In Figure 7, the module and phase impedance sensor are shown when the moisture values range from 30 to $80 \% \mathrm{RH}$ at 2 $\mathrm{kHz}$. In addition, values from the experimental error are presented in the graphs. The common behavior in the three cases is that the module impedance decreases when the humidity increases, whereas the phase impedance is also increased. This fact denotes the capacitance behavior of the sensor at lower moisture values and resistive behavior at higher moisture values, mainly in cotton and medical substrates. Specifically, the impedance module ranges from $13.3 \mathrm{M} \Omega$ to $0.99 \mathrm{M} \Omega$ on $\mathrm{CO}$ substrate and from $3.86 \mathrm{M} \Omega$ to $4 \mathrm{k} \Omega$ on MED substrate. Polyester sensor substrate module impedance has a shorter range of values which are between 14.7 $\mathrm{M} \Omega$ to $6.33 \mathrm{M} \Omega$. In phase impedance graph is observed how the cotton substrates (CO and MED) have the reduction from $-71^{\circ}$ to $-10^{\circ}$, meanwhile the PES substrate sensor is maintaining its capacitive nature with phase values between $-72^{\circ}$ and $-90^{\circ}$. These results denote that the cotton substrates are affected in a higher way for the humidity and this provokes that the module impedance decreases very rapidly and the phase tendency tends to 0 degrees. As a result, the sensor becomes more resistive. For PES substrate sensor the module impedance decreases with a constant slope, and a phase impedance remains almost constant which shows the capacitive behavior along the relative humidity range. Moreover, the error values demonstrate the higher repeatability of PES sensors in front of CO and MED sensors. 

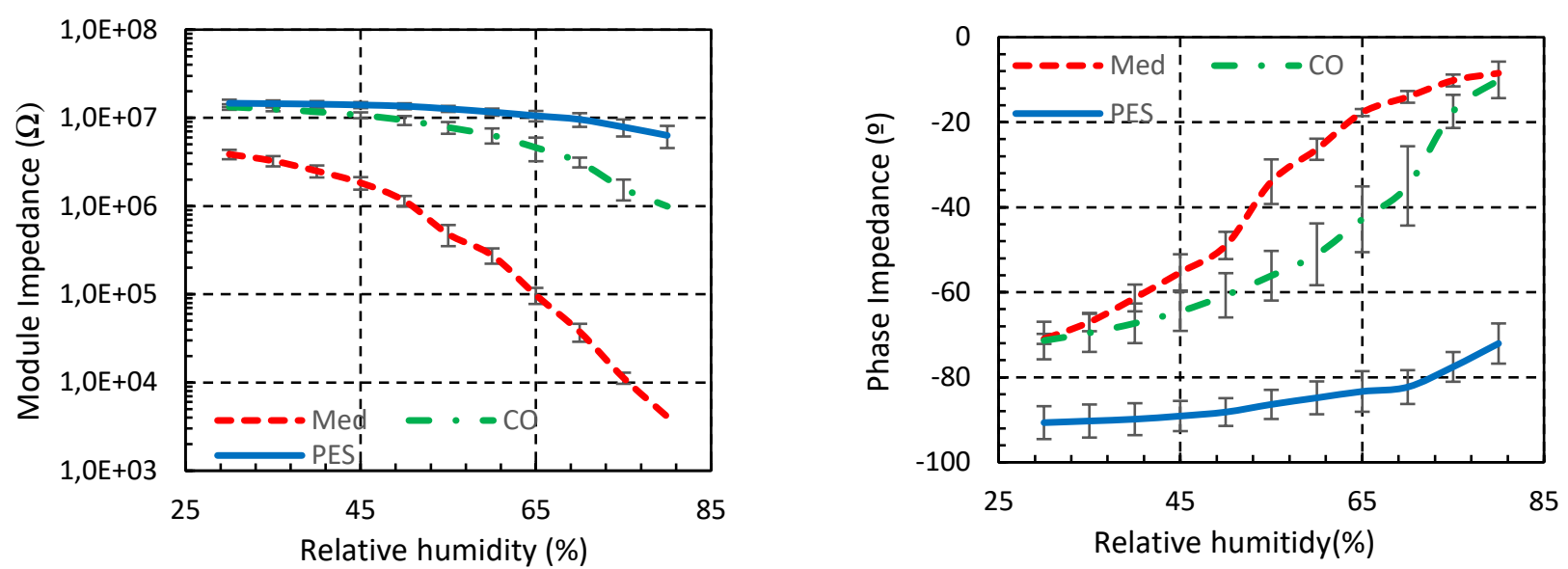

Figure 7. Measured impedance along relative humidity at $2 \mathrm{kHz}$.

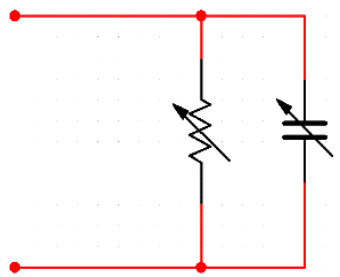

Figure 8. Proposed electrical model for the studied sensor.

The experimental impedance masurement results show that regardless of substrate material the proposed sensor can be modelled by means of a parallel RC circuit, as shown in Figure 8. In the Figure 9 the values for the measured parallel resistance and capacitance are shown at $2 \mathrm{kHz}$ for each studied substrate when the moisture values range from 30 to $80 \% \mathrm{RH}$. It is observed that the resistance is reduced whereas the capacitance increases with the moisture. This fact is due to water molecular build-up on the textile. Therefore, the hydrophilic properties of the material define the electric sensor behavior. In PES the sensor impedance is mainly capacitive, with values lower than $10 \mathrm{pF}$. However, for both cotton substrates (CO and MED) at lower moisture values the impedance is mainly capacitive, with values up to $7 \mathrm{pF}$ and $12 \mathrm{pF}$ for CO and MED, respectively. At higher moisture values the behavior of cotton sensors are mainly resistive with a value up to $450 \mathrm{k} \Omega$ and $1.9 \mathrm{k} \Omega$ for $\mathrm{CO}$ and MED, respectively. These values can be observed and compared on Figure 9.
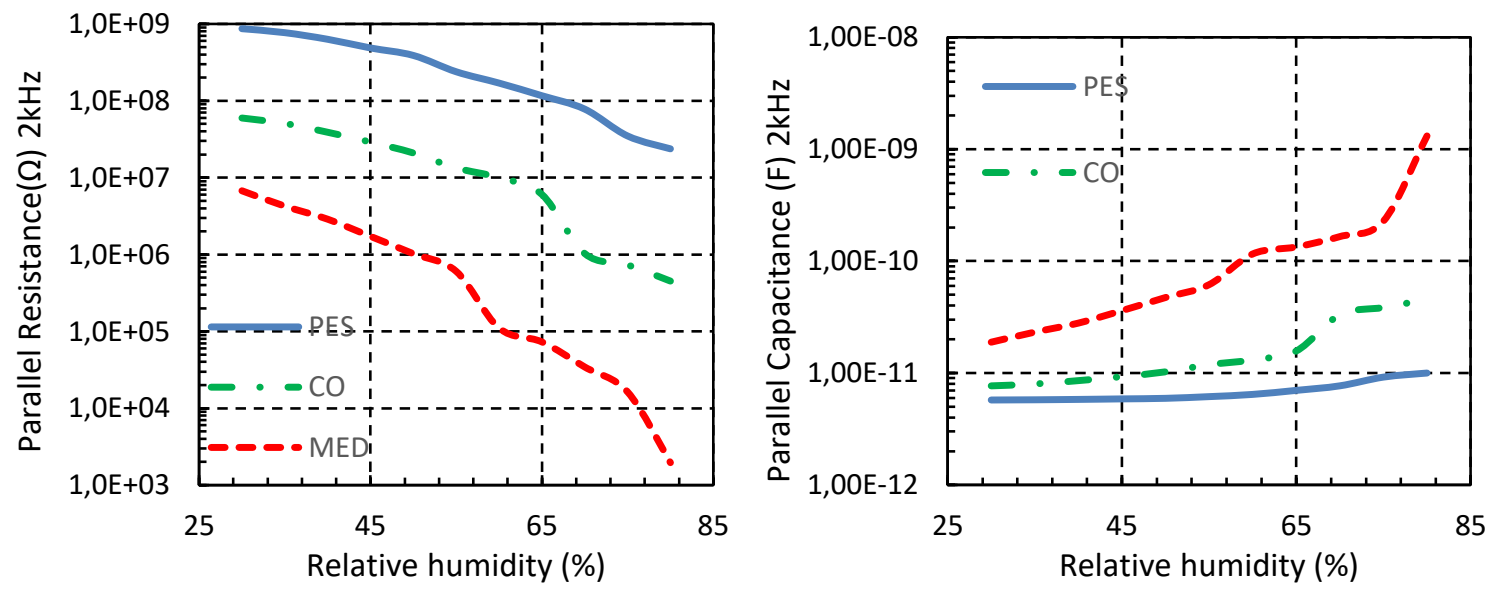

Figure 9. Measured equivalent circuit for the sensor at $2 \mathrm{kHz}$. 
In order to explain the obtained experiments behavior, the relative permittivity has been characterized as detailed in section 2. It is known that the capacitance is related directly with the real part of the permittivity. Figure 10 shows the measured relative permittivity along a small swept of relative humidity. It can be seen that relative permittivity increases with humidity level. Permittivity, for cotton and medical cotton substrates, is very similar, 1.83 and 1.91 at $40 \% \mathrm{RH}$ respectively. On the other side, the polyester has a lower permittivity than both cotton substrates, 1.65 at $40 \% \mathrm{RH}$. If the moisture dependence of the permittivity is analyzed, an enhancement value of $15.4 \%, 15.2 \%$ and $6.6 \%$ it is observed for MED, CO and PES respectively, meanwhile the capacitance increases 491\%, 288\% and 32\% for MED, CO and PES. As a consequence, the capacitance shift cannot be only attributed to permittivity moisture dependence. A reason to explain the obtained behavior is the geometric shift of the sensor. In fact, the dimensions of the sensor are changed due to the increase of the yarn thickness when water molecules are absorbed by the textile. This effect is more present on cotton than polyester fabric and this is why the cotton substrate shows a higher impedance shift.

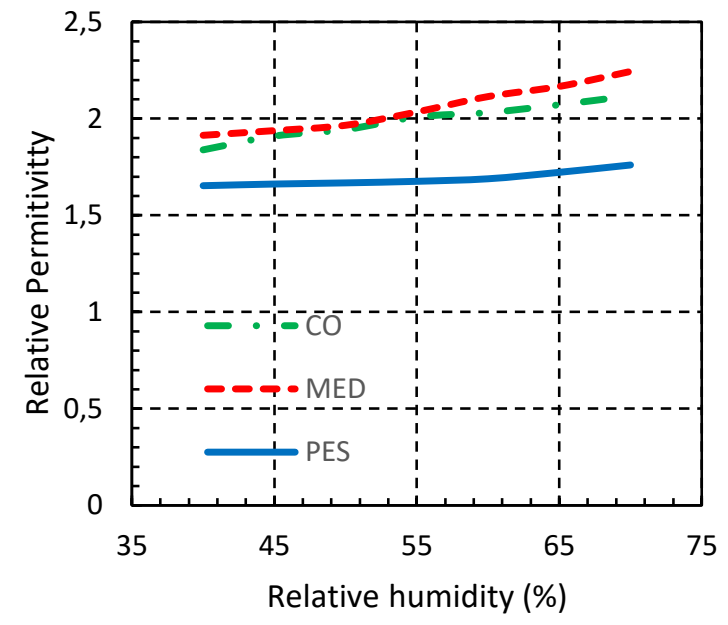

Figure 10. Relative permittivity measured for each substrate.

\section{Conclusion}

In this work, several interdigitated embroidered textile sensors have been proposed and characterized. The sensors have been embroidered over three different substrates, cotton woven fabric, medical cotton woven fabric and polyester woven fabric. The results show that sensor impedance is reduced with the moisture increase. However, in contrast to other moisture sensors, the impedance is not only affected by the relative permittivity shift, but also by the modification of the sensor dimensions due to water molecular absorption. Due to this behavior, the sensors based on cotton fabrics shows an impedance reduction of $99.87 \%$ and $93.23 \%$ for MED and CO, respectively. For polyester substrate an impedance reduction of 56.94\% is achieved. Moreover, the higher concentration of molecular water on cotton fabrics modifies the sensor nature, which shifts from capacitive for low moisture to resistive for high moisture values. This effect is not observed on polyester substrate where the capacitive property remains along all the moisture range. The observed properties can be used as guidelines to define the optimum fabric substrate to be used depending on the sensor target application.

The differences in behaviour can determine the applications where each one will be used. On one side, cotton fabric sensor (including medical) can be used on applications where a quick reaction would be needed but the use of the sensor is not thought to last for long periods of time, such as water leakage or urinate detection. On the other side, polyester fabric sensor can be used on applications where all the range need to be cover and would be working longer periods of time, due to the propertie that polyester has that give the possibilitie to dry faster than cotton, such as sweating sensor applications. 


\section{Acknowledgements}

This work was supported by Spanish Government-MINECO under Project TEC2016-79465-R and Terrassa's city council

"Beques de Recerca per a estudiants de centres universitars amb seu a Terrassa".

\section{References}

[1] I. G. Trindade, F. Martins, R. Dias, C. Oliveira, and J. Machado Da Silva, "Novel textile systems for the continuous monitoring of vital signals: Design and characterization,” Proc. Annu. Int. Conf. IEEE Eng. Med. Biol. Soc. EMBS, vol. 2015Novem, pp. 3743-3746, 2015.

[2] B. Babusiak, S. Borik, and L. Balogova, "Textile electrodes in capacitive signal sensing applications," Meas. J. Int. Meas. Confed., vol. 114, no. March 2017, pp. 69-77, 2018.

[3] J.-F. Wu et al., "Human Limb Motion Detection with Novel Flexible Capacitive Angle Sensor Based on Conductive Textile," electronics, vol. 7, no. 192, 2018.

[4] H. Baskan et al., "Running functional sport vest and short for e-textile applications," IOP Conf. Ser. Mater. Sci. Eng., vol. 254, no. 7, pp. 1-8, 2017.

[5] G. Tartare, X. Zeng, and L. Koehl, "Development of a wearable system for monitoring the firefighter's physiological state," in 2018 IEEE Industrial Cyber-Physical Systems (ICPS), 2018, pp. 561-566.

[6] Y. Chen et al., "Design of High-Performance Wearable Energy and Sensor Electronics from Fiber Materials," ACS Appl. Mater. Interfaces, vol. 11, no. 2, pp. 2120-2129, 2019.

[7] M. Tessarolo, I. Gualandi, and B. Fraboni, "Recent Progress in Wearable Fully Textile Chemical Sensors," Adv. Mater. Technol., vol. 3, no. 10, p. 1700310, Oct. 2018.

[8] L. Areekath et al., “An Electric-Field Based Breathing Rate Monitor,” in 2018 IEEE International Symposium on Medical Measurements and Applications (MeMeA), 2018.

[9] K. Qi et al., "A Highly Stretchable Nanofiber-Based Electronic Skin with Pressure-, Strain-, and Flexion-Sensitive Properties for Health and Motion Monitoring," ACS Appl. Mater. Interfaces, vol. 9, no. 49, pp. 42951-42960, 2017.

[10] J. Eom et al., "Highly Sensitive Textile Strain Sensors and Wireless User-Interface Devices Using All-Polymeric Conducting Fibers,” ACS Appl. Mater. Interfaces, vol. 9, no. 11, pp. 10190-10197, 2017.

[11] L. M. Castano and A. B. Flatau, "Smart fabric sensors and e-textile technologies: A review," Smart Mater. Struct., vol. 23, no. 5, 2014 .

[12] T. Grethe, S. Borczyk, K. Plenkmann, M. Normann, M. Rabe, and A. Schwarz-Pfeiffer, "Textile humidity sensors," Symp. Des. Test, Integr. Packag. MEMS/MOEMS, DTIP 2018, pp. 1-3, 2018.

[13] M. Martinez-Estrada et al., "Embroidery Textile Moisture Sensor," Proceedings, vol. 2, no. 13, p. 1057, Nov. 2018.

[14] EURATEX, “EURATEX Annual report 2017," 2017.

[15] INDITEX, "INDITEX Annual report 2017,” 2018.

[16] M. Martínez-Estrada, B. Moradi, R. Fernández-Garcia, and I. Gil, "Impact of Conductive Yarns on an Embroidery Textile Moisture Sensor," Sensors, vol. 19, no. 5, p. 1004, 2019.

[17]BERNADETT CSASZAR. (2019). Embroidery Machine vs Sewing Machine. Hatch by Wilcom. https://www.wilcom.com/Hatch/Testimonials/ArtMID/16456/ArticleID/617/Embroidery-Machine-vs-Sewing-Machine.aspx

[18] A. Devices, “AD5933 1 MSPS, 12-Bit Impedance Converter, Network Analyzer.” .

Word Count: 2910 